\title{
A Fast Beamforming Method to Localize an Acoustic Emission Source under Unknown Wave Speed
}

\author{
Junfei Tai ${ }^{1}$, Tian He ${ }^{1}$, Qiang Pan ${ }^{1}$, Dayi Zhang ${ }^{2, *}$ and Xiaoran Wang ${ }^{1}$ \\ 1 School of Transportation Science and Engineering, Beihang University, Beijing 100083, China; \\ taijunfei@buaa.edu.cn (J.T.); hetian@buaa.edu.cn (T.H.); panqiang@buaa.edu.cn (Q.P.); \\ wangxiaoran@buaa.edu.cn (X.W.) \\ 2 School of Energy and Power Engineering, Beihang University, Beijing 100083, China \\ * Correspondence: dayi@buaa.edu.cn; Tel.: +86-134-2616-6834
}

Received: 20 January 2019; Accepted: 28 February 2019; Published: 4 March 2019

\begin{abstract}
The beamforming method is capable of localizing the acoustic emission source in a large-scale structure but its accuracy relies strongly on the assumed propagation speed and it is quite time consuming to apply in online monitoring. This paper proposes a fast beamforming method to localize an acoustic emission source in a thin-walled structure with unknown wave speed. Firstly, the Bartlett beamforming method (BBM) is introduced into broadband Lamb wave signal processing to develop an L-shape array-based damage source localization method for a thin-walled structure. Secondly, the fast Bartlett beamforming method (FBBM) is proposed based on the characteristics of BBM. Finally, the pencil-lead break test is carried out to validate the proposed method. The test results show that the FBBM can accurately localize the damage source by any given probable wave speed much more rapidly than traditional delay-and-sum beamforming.
\end{abstract}

Keywords: acoustic emission; Bartlett beamforming; fast damage localization; structure health monitoring

\section{Introduction}

Ultrasonic techniques, including active and passive sensing, have been widely studied and applied in the field of structure health monitoring [1,2]. Passive ultrasonics, commonly referred to as acoustic emission (AE), has been extensively studied in real time damage monitoring. For example, Kundu et al. [3] proposed an AE source localization method based on Bayesian identification; they trained a probabilistic model to give probabilistic descriptors of damage locations in composites structures. Sikdar et al. [4,5] concentrated on the identification and monitoring problems of a damage-induced AE source in the advanced sandwich composite structure, and they proposed systematic methods based on an evolutionary algorithm and a localization algorithm to identify structure damage.

Damage localization based on AE technology is critical for damage detection and health evaluation, and this has led to a variety of applications [6]. Among many AE source localization technologies, the time difference of arrivals (TDOA) method has become the most widely studied and applied one in practical applications [7]. However, its inherent property makes the accuracy of the TDOA method highly sensitive to time difference and wave speed [8]. Therefore, many scholars concentrated on obtaining accurate time difference or accurate wave velocity. For instance, Ziola et al. [9] used the cross-correlation technique to study the characteristics of stress waves between special mode frequency and wave velocity. Hamstad et al. [10] determined the arrival time of signals by means of wavelet transform. Carpinteri [11] used the Akaike Information Criterion to recognize the arrival time of $\mathrm{AE}$ waves in concrete. In order to improve the localization accuracy, localization methods without wave speed have been studied. For example, Kundu et al. [12] studied the mathematical model 
based on the triangulation technique and developed a method without considering wave speed in the fields of damage localization for metal and composite materials [13]. Hensman et al. [14] proposed a time-reversal AE source localization method combined with statistical theory for structures with holes.

Although the acoustic emission localization methods have been extensively studied, there is still a great deal of work to be done [15]. The beamforming localization method is one of the promising attempts. McLaskey [16] and He et al. [17] introduced the beamforming method (BFM) to localize an AE source, which is easy to implement and exhibits high localization accuracy in a noisy environment [18]. Xiao et al. [19] found that the localization result of a uniform linear array of sensors is sensitive to wave speed only in the direction perpendicular to the linear array. Hence, they used two orthogonal linear arrays to determine the horizontal and vertical coordinates respectively and the results show that this is effective for source localization under inaccurate wave speed. Although BFM shows high localization precision and great robustness, its inherent drawback is its low computational efficiency. In other words, the more sensors used, the slower the calculation speed.

This paper aims to develop a fast and reliable localization method for AE sources on a thin-walled structure based on the Bartlett beamforming method (BBM) [20], no matter whether the wave speed is accurate or not. Since the method completely ignores the localization performance perpendicular to the array direction in exchange for consistency and accuracy of localizing effects parallel to the array direction, it can localize the AE source with high speed and precision by an L-shaped sensors array.

\section{Principles}

A frequency-domain weighted beamforming, named BBM, is introduced to establish the fast localization method. BBM, as a classic beamforming method, has been widely used to localize acoustic sources [20]. The schematic diagram of Bartlett beamforming in a plate-like structure is shown in Figure 1. For a two-dimensional plane in which $M$ pieces of sensors are linearly arranged as shown in Figure 1, the signals $\mathbf{X}(\mathbf{t})=\left[x_{1}(t), x_{2}(t), \cdots, x_{M}(t)\right]^{T}$ received by sensor array from the near-field AE source can be expressed by:

$$
\mathbf{X}(\mathbf{t})=a(x, y, f) S(t)+\mathbf{N}(\mathbf{t})
$$

where $\mathbf{N}(\mathbf{t})$ is the noise received by $M$ sensors of the array and can be given by $\mathbf{N}(\mathbf{t})=\left[n_{1}(t), n_{2}(t), \cdots, n_{M}(t)\right]^{T}$. The function $a(x, y, f)$ is defined as the steering vector, which represents an overall representation of the original signal $S(t)$ transmitted from the position $(x, y)$ under a particular array form:

$$
a(x, y, f)=\left[1, e^{-j 2 \pi f \tau_{2}}, \cdots, e^{-j 2 \pi f \tau_{M}}\right]^{T}
$$

where $f$ is the center frequency of the signal, and $\tau_{i}$ is the time delay of the signal received by the $i$-th sensor relative to the reference signal:

$$
\tau_{i}=\frac{\left|\overrightarrow{r_{i}}\right|-\left|\overrightarrow{r_{1}}\right|}{c}
$$

The signal represented by Equation (1) is processed by the weighting matrix $W^{H}(x, y, f)$. The dimension of $W^{H}(x, y, f)$ is $1 \times N$, and the superscript $H$ represents the conjugate transpose of the matrix $W(x, y, f)$, which can be written by:

$$
W(x, y, f)=\left[W_{1}(x, y, f), W_{2}(x, y, f), \cdots, W_{M}(x, y, f)\right]^{T}
$$

The element of $W(x, y, f)$ may be a complex number whose modulo represents the weight on the amplitude of the output. The signal output after the weighting matrix processing is expressed by:

$$
Y(t)=W^{H}(x, y, f) \mathbf{X}(\mathbf{t})
$$


The signal processed by the weighting matrix is required to have the maximum power, and the mean square value of the signal is used to represent the average power of the signal:

$$
P=E\left[|Y(t)|^{2}\right]=W^{H} E\left[\mathbf{X}(\mathbf{t}) \mathbf{X}^{H}(\mathbf{t})\right] W
$$

where $E[$ ] represents the mean operator. Substituting Equation (1) into Equation (6), the power $P$ can be rewritten by:

$$
P=E\left[|S(t)|^{2}\right]\left(W^{H} a a^{H} W\right)+\sigma^{2}\|W\|^{2}
$$

where $\|\cdot\|$ represents the norm of vector, and $\sigma^{2}$ is the noise variance. When the noise variance is constant, a constraint must be established to ensure the signal to noise ratio of the output not be affected by the weighting matrix:

$$
\|W\|=1
$$

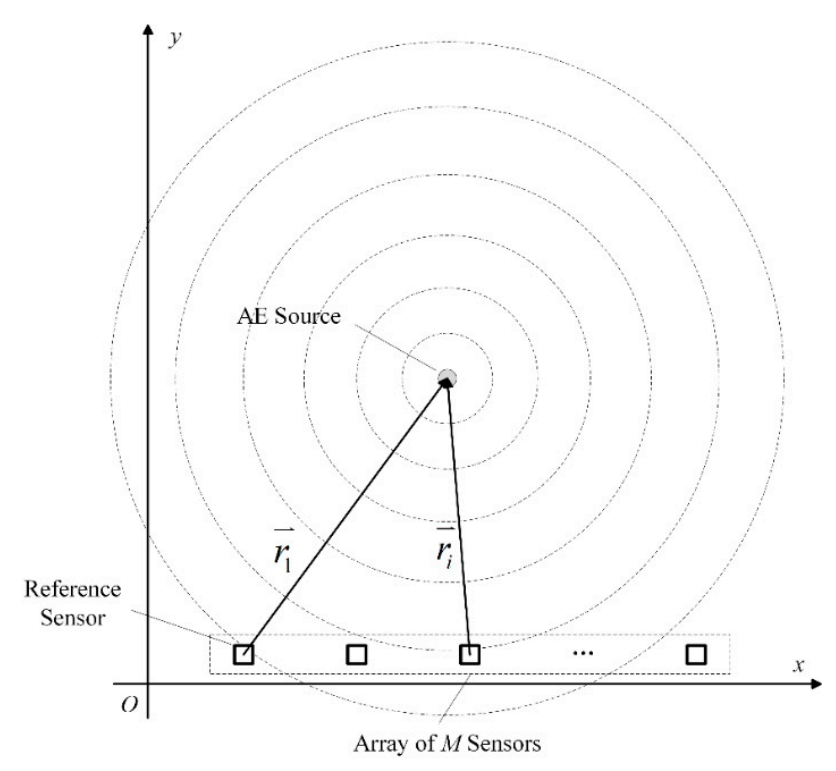

Figure 1. Schematic diagram of the near-field beamforming.

Under the constraint of Equation (8), Lagrange multiplier method is used to solve $W$ to maximize the average signal power:

$$
W=\frac{a(x, y, f)}{\sqrt{a^{H}(x, y, f) a(x, y, f)}}
$$

Substituting Equation (9) into Equation (6), the maximum power $P$ can be reached:

$$
P=\frac{a^{H}(x, y, f) R a(x, y, f)}{a^{H}(x, y, f) a(x, y, f)}
$$

where $R=E\left[\mathbf{X}(\mathbf{t}) \mathbf{X}^{\mathbf{H}}(\mathbf{t})\right]$ is obtained by calculating the covariance matrix of the array signals. R has no relationship with the assumed wave speed, while it is affected by the propagation speed in the specific material. Because the change of propagation speed will affect the received signals $\mathbf{X}(\mathbf{t})$, and the change of $\mathbf{X}(\mathbf{t})$ is mainly about the relative phase position of signals from different sensor channels. That is to say, $R$ still contains the information about where the true source is, and the function $a(x, y, f)$ is used to find the most matched point $(x, y)$. For the condition that the dimension of $\mathbf{X}(\mathbf{t})$ is $\mathrm{M} \times \mathrm{T}($ where $\mathrm{T}$ represents the length of the digital signals), $R$ will be a matrix with the dimension of $\mathrm{M} \times \mathrm{M}$. The point $(x, y)$ where the power $P$ reaches maximum, is considered to be the acoustic source. 


\section{Implementation and Test of the Algorithm}

\subsection{Implementation and Improvement of the Proposed Algorithm}

The localization performance of the presented algorithm is tested by simulated signals of damage. The dimension of the thin-walled steel structure in the simulation is $700 \times 700 \times 5 \mathrm{~mm}$. Density, elastic modulus, and the Poisson's ratio of material are set as $7.8 \times 10^{3} \mathrm{~kg} / \mathrm{m}^{3}, 2.09 \times 10^{11} \mathrm{~N} / \mathrm{m}^{2}$ and 0.3 , respectively. The damage source was simulated by a pair of transient triangular forces lasting $5 \mu \mathrm{s}$, and the time profile of that is shown in Figure 2. Due to the short wavelength of the AE wave under normal conditions (for example, the bending wave is about $20 \mathrm{~mm}$ and the extending wave is about $35 \mathrm{~mm}$ in the AE wave with center frequency of $150 \mathrm{kHz}$ ), it is sufficient to mesh the geometry model by $1 \times 1 \mathrm{~mm}^{2}$ to simulate the modes of the AE wave in the interested frequency band. The element type used in Abaqus (version 6.14, Dassault Systèmes Simulia Corp, Johnston, RI, USA) is C3D8R, which is a linearly reduced integration element with accurate displacement results and less computation time. Specific simulation details are described and validated in the literature [21].

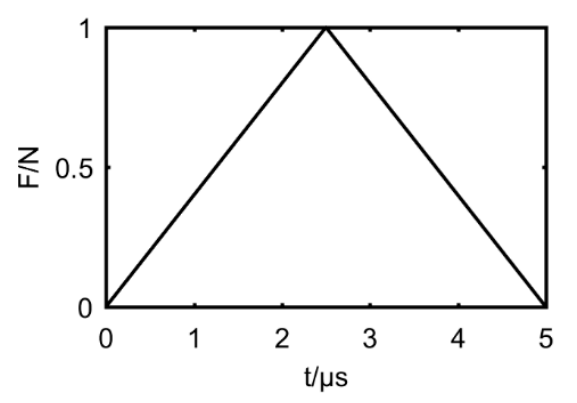

Figure 2. The time profile of transient triangular force.

Figure 3 shows the position of two perpendicular linear sensor arrays in a Cartesian coordinate system. The excitation positions of the damage sources at four different points are plotted in Figure 3.

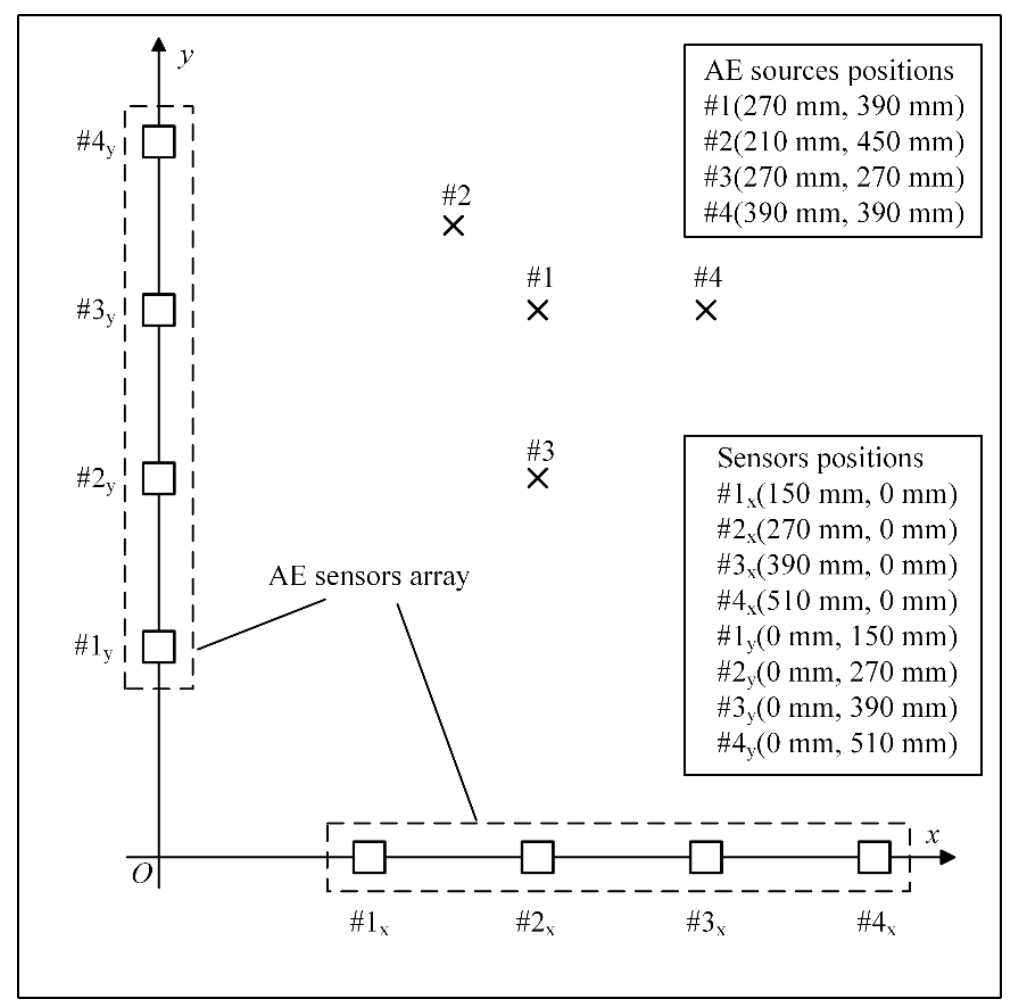

Figure 3. The arrangement of sensors and acoustic emission (AE) sources in the simulation. 
Due to the dispersion and energy attenuation phenomena of the propagating AE wave, the excited transient triangular wave expands to a broadband signal through a complex propagation process. The time-domain waveform and frequency spectrum of the signal after propagating about $400 \mathrm{~mm}$ with a center frequency of $150 \mathrm{kHz}$ are shown in Figure 4.

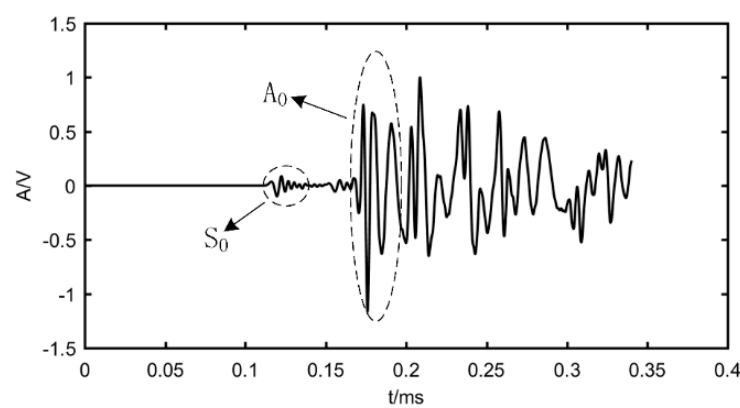

(a)

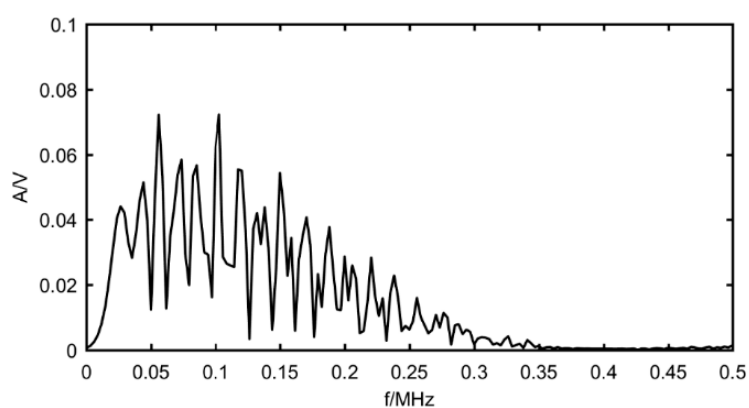

(b)

Figure 4. The AE signal acquired by simulation: (a) waveform of signal; (b) frequency spectrum.

The localization performed by the $S_{0}$ wave is shown in Figure $4 \mathrm{a}$. The $S_{0}$ wave is a symmetrical mode wave with low dispersion and good waveform consistency when it propagates far away from the source. The preliminary localization results of one set of data by using the proposed algorithm is shown in Figure 5.

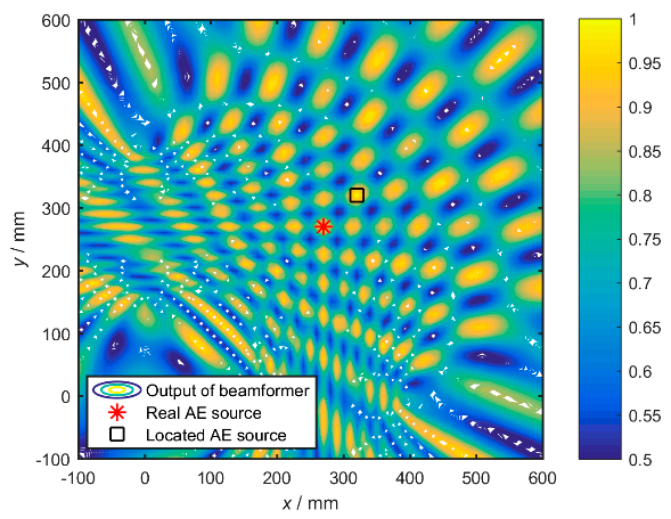

Figure 5. The preliminary localization determined by the $S_{0}$ wave.

As can be seen from Figure 4, the localization effect is not satisfying. It means that even if the dispersion of the $S_{0}$ wave is not severe, the broadband AE signal cannot be used by Bartlett beamforming to localize sources effectively. Therefore, the solution method is presented by decomposing the broadband signal into several narrowband signals in the frequency domain, then using the Bartlett beamforming described in the second section to estimate the power spectrum on each sub-band, and finally summing them all to reach the overall result. The steering vector of the sub-band signal can be written as:

$$
a\left(x, y, f_{i}\right)=\left[1, e^{-j 2 \pi f_{i} \tau_{2}}, \cdots, e^{-j 2 \pi f_{i} \tau_{M}}\right]^{T}
$$

where $f_{\mathrm{i}}$ is the center frequency of the $i$-th sub-band. Therefore, the weighting matrix described by Equation (9) and the power $P$ described by Equation (10) can be generalized by:

$$
W_{i}=\frac{a\left(x, y, f_{i}\right)}{\sqrt{a^{H}\left(x, y, f_{i}\right) a\left(x, y, f_{i}\right)}}
$$




$$
P=\sum_{i=1}^{n} P_{i}=\sum_{i=1}^{n} \frac{a^{H}\left(x, y, f_{i}\right) R a\left(x, y, f_{i}\right)}{a^{H}\left(x, y, f_{i}\right) a\left(x, y, f_{i}\right)}
$$

where $n$ is the number of sub-bands decomposed by the broadband signals.

The localizations result of four sets of signals is recalculated according to the improved algorithm and shown in Figure 6. The yellow lines represent the area with high calculated power $P$. It shows the case where the assumed propagation speed is $5300 \mathrm{~m} / \mathrm{s}$. Obviously, the localization results are quite accurate, and the improved broadband Bartlett beamforming has very good applicability in processing broadband AE signals.

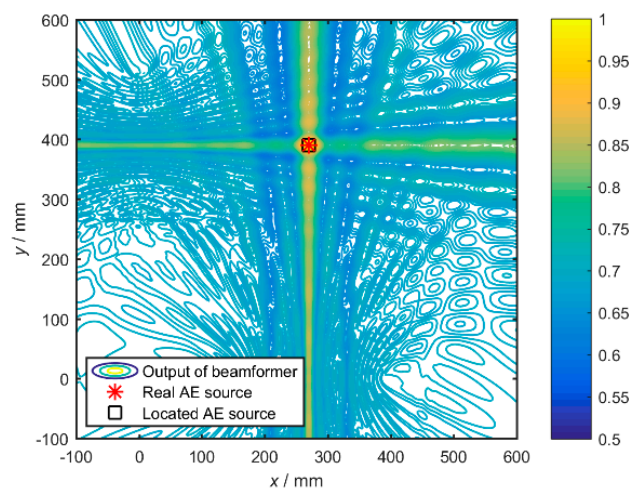

(a)

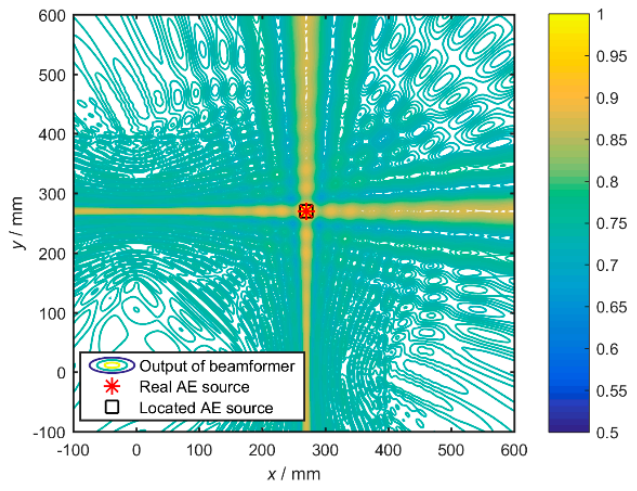

(c)

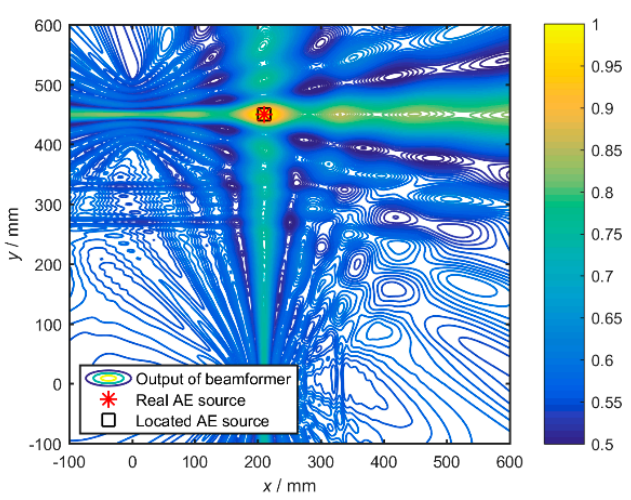

(b)

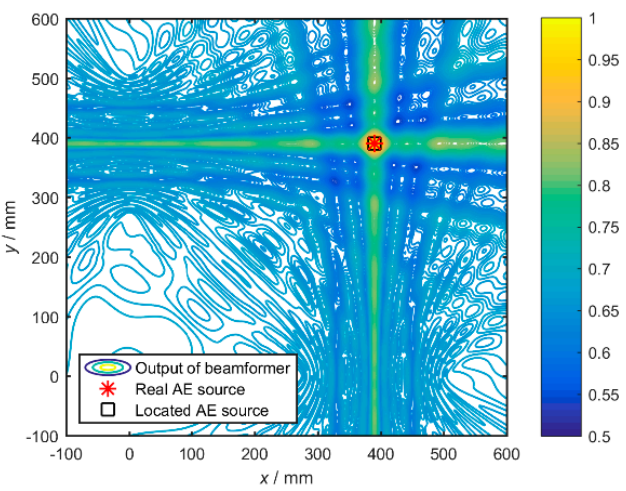

(d)

Figure 6. The localization results of four sets of AE sources: (a) Set 1; (b) Set 2; (c) Set 3; (d) Set 4 .

In order to further evaluate the algorithm with inaccurate propagation speed, the assumed speed ranging from $2000 \mathrm{~m} / \mathrm{s}$ to $12,000 \mathrm{~m} / \mathrm{s}$ is used to localize with an interval of $2000 \mathrm{~m} / \mathrm{s}$. The results are summarized in Table 1. It can be seen that the proposed algorithm can accurately localize the sources even with inaccurate propagation speeds.

Table 1. Localization results of Bartlett beamforming under inaccurate wave speed.

\begin{tabular}{|c|c|c|c|c|c|c|c|c|}
\hline \multirow{4}{*}{ Speed $\left(m \cdot s^{-1}\right)$} & \multicolumn{8}{|c|}{ Location } \\
\hline & \multicolumn{8}{|c|}{ AE Sources $(\mathrm{mm}, \mathrm{mm})$} \\
\hline & \multicolumn{2}{|c|}{ Set 1} & \multicolumn{2}{|c|}{ Set 2} & \multicolumn{2}{|c|}{ Set 3} & \multicolumn{2}{|c|}{ Set 4} \\
\hline & $\begin{array}{c}x \\
270\end{array}$ & $\begin{array}{c}y \\
390\end{array}$ & $\begin{array}{c}x \\
210\end{array}$ & $\begin{array}{c}y \\
450\end{array}$ & $\begin{array}{c}x \\
270\end{array}$ & $\begin{array}{c}y \\
270\end{array}$ & $\begin{array}{c}x \\
390\end{array}$ & $\begin{array}{c}y \\
390\end{array}$ \\
\hline 2000 & 270 & 390 & 210 & 450 & 270 & 270 & 390 & 390 \\
\hline 4000 & 270 & 390 & 210 & 450 & 270 & 270 & 390 & 390 \\
\hline 6000 & 270 & 390 & 210 & 450 & 270 & 270 & 390 & 390 \\
\hline 8000 & 270 & 390 & 210 & 450 & 270 & 270 & 390 & 390 \\
\hline 10,000 & 270 & 390 & 210 & 450 & 270 & 270 & 390 & 390 \\
\hline 12,000 & 270 & 390 & 210 & 450 & 270 & 270 & 390 & 390 \\
\hline
\end{tabular}




\subsection{Principle of the Fast Algorithm}

A three-dimensional localization diagram is shown in Figure 7, from which the beam characteristics of the proposed method can be observed. The array directions marked by white arrows are the location of linear sensor arrays. There are two beams perpendicular to the two arrays respectively, and the power of the entire beam is higher than the power of other regions. Therefore, one beam can clearly localize one coordinate of the AE source in the array direction, which means that the two perpendicular beams are able to determine the position of the AE source. However, there is no need to find out the complete two beams; the marginal profile (Sidebands) which is perpendicular to the beams is enough to localize the source position. It can greatly increase the localization efficiency. The flowcharts of BBM and FBBM are compared in Figure 8.

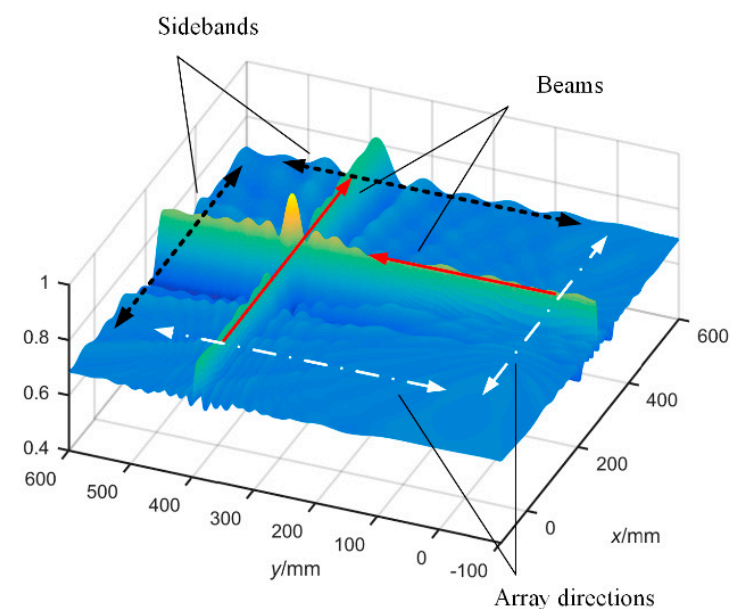

Figure 7. The three-dimension beamforming output diagram of a set of simulated signals.

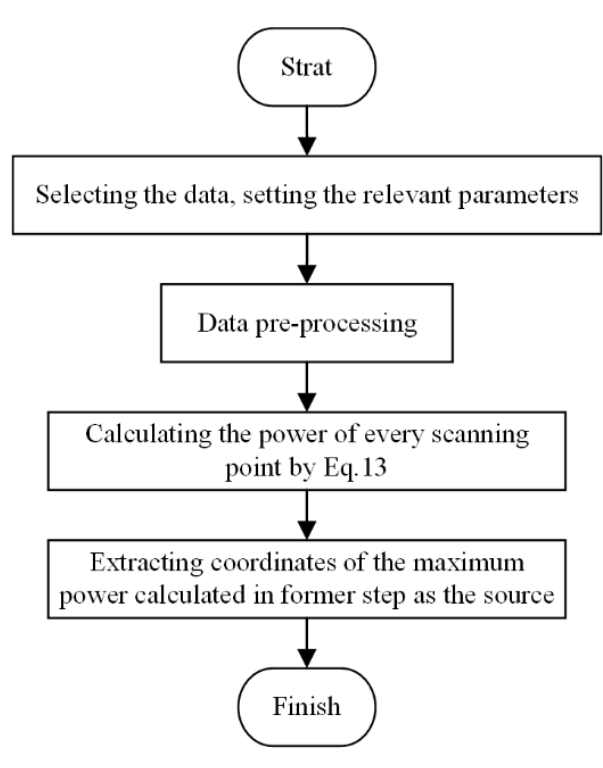

(a)

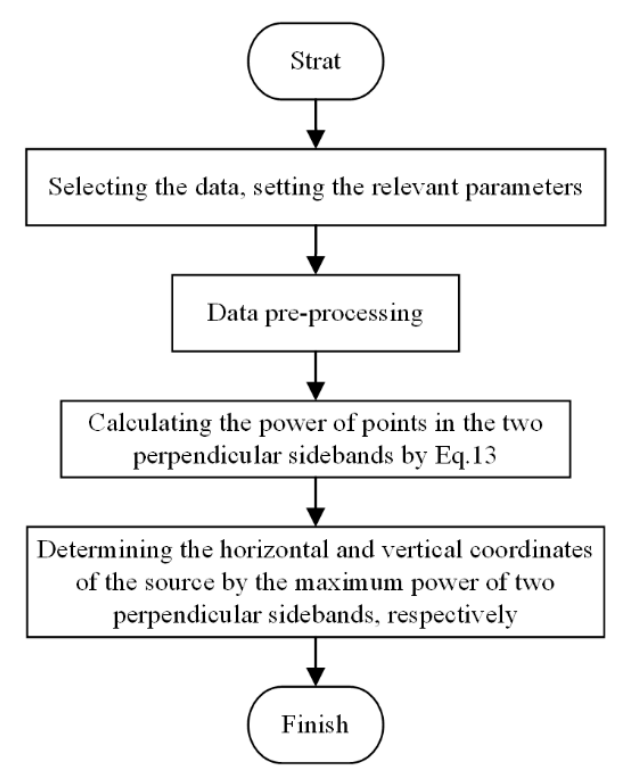

(b)

Figure 8. The flowcharts of the Bartlett beamforming method (BBM) and fast Bartlett beamforming method (FBBM): (a) flowchart of BBM; (b) flowchart of FBBM.

A comparison of Bartlett beamforming and fast Bartlett beamforming under the $1 \mathrm{~mm}$ scanning grid is conducted at propagation speed $5300 \mathrm{~m} / \mathrm{s}$. The average calculation times of two algorithms are measured by MATLAB in the same computer under the same condition. The results are shown in 
Table 2. It can be seen from Table 2 that the calculation speed of the fast Bartlett beamforming method (FBBM) is significantly higher than that of BBM while maintaining the same positioning accuracy.

Table 2. Localization comparison of the BBM and the FBBM at $\mathrm{v}=5300 \mathrm{~m} / \mathrm{s}$.

\begin{tabular}{|c|c|c|c|c|c|c|c|c|c|}
\hline \multirow{3}{*}{ Results } & \multicolumn{8}{|c|}{ AE Sources $(\mathrm{mm}, \mathrm{mm})$} & \multirow{3}{*}{ Average Time (s) } \\
\hline & \multicolumn{2}{|c|}{ Set 1} & \multicolumn{2}{|c|}{ Set 2} & \multicolumn{2}{|c|}{ Set 3} & \multicolumn{2}{|c|}{ Set 4} & \\
\hline & $\begin{array}{c}x \\
270\end{array}$ & $\begin{array}{c}y \\
390\end{array}$ & $\begin{array}{c}x \\
210\end{array}$ & $\begin{array}{c}y \\
450\end{array}$ & $\begin{array}{c}x \\
270\end{array}$ & $\begin{array}{c}y \\
270\end{array}$ & $\begin{array}{c}x \\
390\end{array}$ & $\begin{array}{c}y \\
270\end{array}$ & \\
\hline BBM & 270 & 390 & 210 & 450 & 270 & 270 & 390 & 270 & 224.38 \\
\hline FBBM & 270 & 390 & 210 & 450 & 270 & 270 & 390 & 270 & 0.72 \\
\hline
\end{tabular}

The performance of fast Bartlett beamforming under different propagation speeds is calculated, and the results in Table 3 show that the fast Bartlett beamforming is as accurate as Bartlett beamforming under the propagation speeds ranging from $2000 \mathrm{~m} / \mathrm{s}$ to $12,000 \mathrm{~m} / \mathrm{s}$.

Table 3. Localization results of fast Bartlett beamforming under inaccurate wave speed.

\begin{tabular}{|c|c|c|c|c|c|c|c|c|}
\hline \multirow{4}{*}{ Speed $\left(m \cdot s^{-1}\right)$} & \multicolumn{8}{|c|}{ Location } \\
\hline & \multicolumn{8}{|c|}{ AE Sources (mm, mm) } \\
\hline & \multicolumn{2}{|c|}{ Set 1} & \multicolumn{2}{|c|}{ Set 2} & \multicolumn{2}{|c|}{ Set 3} & \multicolumn{2}{|c|}{ Set 4} \\
\hline & $\begin{array}{c}x \\
270\end{array}$ & $\begin{array}{c}y \\
390\end{array}$ & $\begin{array}{c}x \\
210\end{array}$ & $\begin{array}{c}y \\
450\end{array}$ & $\begin{array}{c}x \\
270\end{array}$ & $\begin{array}{c}y \\
270\end{array}$ & $\begin{array}{c}x \\
390\end{array}$ & $\begin{array}{c}y \\
390\end{array}$ \\
\hline 2000 & 270 & 390 & 210 & 450 & 270 & 270 & 390 & 390 \\
\hline 4000 & 270 & 390 & 210 & 450 & 270 & 270 & 390 & 390 \\
\hline 6000 & 270 & 390 & 210 & 450 & 270 & 270 & 390 & 390 \\
\hline 8000 & 270 & 390 & 210 & 450 & 270 & 270 & 390 & 390 \\
\hline 10,000 & 270 & 390 & 210 & 450 & 270 & 270 & 390 & 390 \\
\hline 12,000 & 270 & 390 & 210 & 450 & 270 & 270 & 390 & 390 \\
\hline
\end{tabular}

\section{Experimental Verification}

To further validate the algorithm performance, the experimental data in the literature by Xiao et al. [19] is used in this study and the result is compared with the localization method in that literature. Xiao et al. [19] used a pencil-lead break (PLB) test to generate a Hsu-Nielsen source and localized the sources by delay-and-sum beamforming. The PLB test is carried out on a homogeneous thin-walled steel plate with the size $700 \times 700 \times 5 \mathrm{~mm}$. The layout of the L-shaped array and eight AE sources are shown in Figure 9. The sampling frequency is $3 \mathrm{MHz}$ and the waveform of a set of experimental data compared with simulated data is shown in Figure 10. This indicates that the simulation signal has the main characteristic of the experimental AE signal.

Based on the collected data of 8 sources, the $S_{0}$ waves in the signals were used for localization at different speeds, which is summarized in Table 4 in comparison with the paper [19]. It can be seen that the localization performance of fast Bartlett beamforming is generally better than that of delay-and-sum beamforming at the same propagation speed, and it can be applied to a wider range of propagation speeds with good stability. In addition, the calculation time of delay-and-sum beamforming and fast Bartlett beamforming under different scanning accuracy is compared. The calculation time is averaged by 8 sets of data at the same wave speed $v=5300 \mathrm{~m} / \mathrm{s}$, which is shown in Table 5 . As can be seen from Table 5 , the calculation of fast Bartlett beamforming under different scanning accuracy is much faster than delay-and-sum beamforming by about $50-420$ times. 


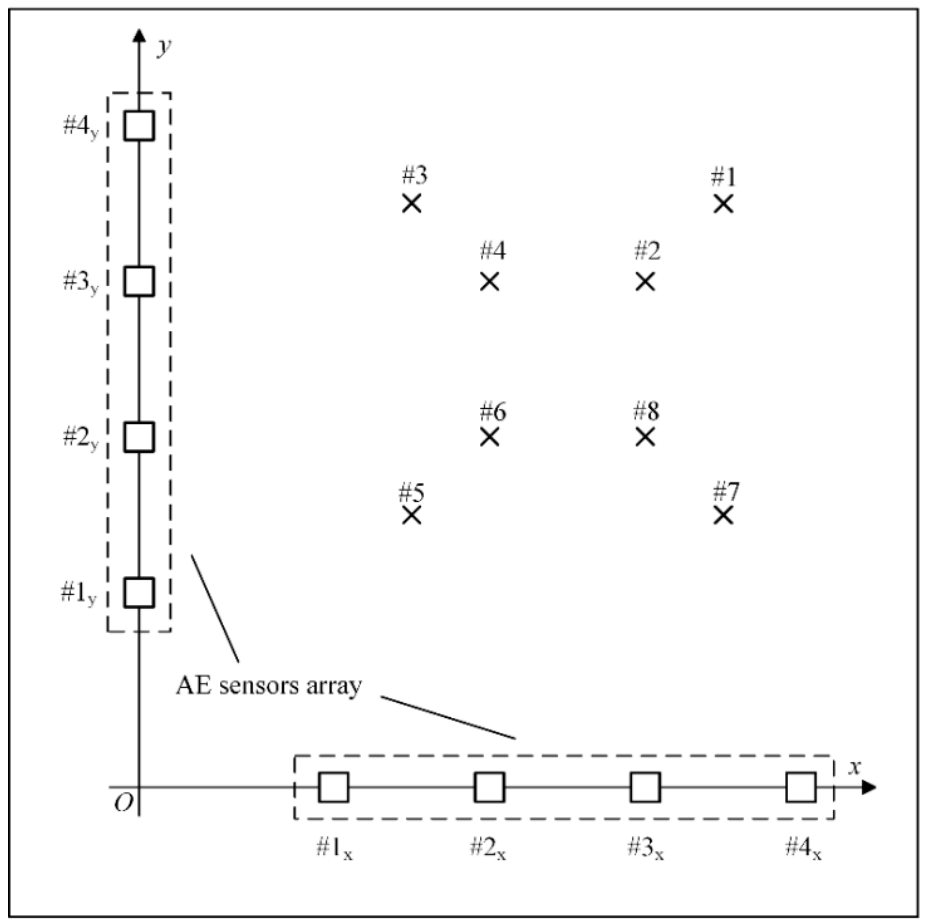

$\mathrm{AE}$ sources positions $\# 1(450 \mathrm{~mm}, 450 \mathrm{~mm})$ $\# 2(390 \mathrm{~mm}, 390 \mathrm{~mm})$ \#3 $(210 \mathrm{~mm}, 450 \mathrm{~mm})$ $\# 4(270 \mathrm{~mm}, 390 \mathrm{~mm})$ $\# 5(210 \mathrm{~mm}, 210 \mathrm{~mm})$ $\# 6(270 \mathrm{~mm}, 270 \mathrm{~mm})$ \#7 $(450 \mathrm{~mm}, 210 \mathrm{~mm})$ $\# 8(390 \mathrm{~mm}, 270 \mathrm{~mm})$

Sensors positions

\#1 $(150 \mathrm{~mm}, 0 \mathrm{~mm})$

$\# 2 \times(270 \mathrm{~mm}, 0 \mathrm{~mm})$

\#3 $3(390 \mathrm{~mm}, 0 \mathrm{~mm})$

$\# 4_{\mathrm{x}}(510 \mathrm{~mm}, 0 \mathrm{~mm})$

\#1 1 (0 mm, $150 \mathrm{~mm})$

\#2 $(0 \mathrm{~mm}, 270 \mathrm{~mm})$

$\# 3 \mathrm{y}(0 \mathrm{~mm}, 390 \mathrm{~mm})$

$\# 4_{y}(0 \mathrm{~mm}, 510 \mathrm{~mm})$

Figure 9. The arrangement of sensors and AE sources in the pencil-lead break (PLB) test.

Table 4. Localization results comparison by PLB test.

\begin{tabular}{|c|c|c|c|c|c|c|c|c|c|}
\hline \multirow{4}{*}{ Categories of Algorithm } & \multirow{4}{*}{$\begin{array}{c}\text { Propagation Speed } \\
\left(\mathrm{m} \cdot \mathrm{s}^{-1}\right)\end{array}$} & \multicolumn{8}{|c|}{ AE Sources $(\mathrm{mm}, \mathrm{mm})$} \\
\hline & & \multicolumn{2}{|c|}{ Set 1} & \multicolumn{2}{|c|}{ Set 2} & \multicolumn{2}{|c|}{ Set 3} & \multicolumn{2}{|c|}{ Set 4} \\
\hline & & $x$ & $y$ & $x$ & $y$ & $x$ & $y$ & $x$ & $y$ \\
\hline & & 450 & 450 & 390 & 390 & 210 & $\stackrel{5}{450}$ & 270 & 390 \\
\hline \multirow{5}{*}{$\begin{array}{c}\text { Delay-and-sum } \\
\text { beamforming [19] }\end{array}$} & 2000 & 440 & 450 & 390 & 390 & 210 & 460 & 260 & 390 \\
\hline & 2500 & 450 & 460 & 390 & 390 & 200 & 460 & 260 & 390 \\
\hline & 3000 & 450 & 460 & 390 & 390 & 200 & 470 & 260 & 390 \\
\hline & 3500 & 450 & 460 & 390 & 420 & 200 & 470 & 260 & 390 \\
\hline & 4000 & 450 & 460 & 390 & 420 & 200 & 470 & 260 & 390 \\
\hline \multirow{9}{*}{ Fast Bartlett beamforming } & 2000 & 450 & 450 & 390 & 390 & 210 & 450 & 270 & 390 \\
\hline & 2500 & 450 & 450 & 390 & 390 & 210 & 450 & 270 & 390 \\
\hline & 3000 & 450 & 450 & 390 & 390 & 210 & 450 & 270 & 390 \\
\hline & 3500 & 450 & 450 & 390 & 390 & 210 & 450 & 270 & 390 \\
\hline & 4000 & 450 & 450 & 390 & 390 & 210 & 450 & 270 & 390 \\
\hline & 6000 & 450 & 450 & 390 & 390 & 210 & 450 & 270 & 390 \\
\hline & 8000 & 450 & 450 & 390 & 390 & 210 & 450 & 270 & 390 \\
\hline & 10,000 & 450 & 450 & 389 & 390 & 210 & 450 & 270 & 390 \\
\hline & 12,000 & 450 & 449 & 390 & 390 & 211 & 449 & 270 & 390 \\
\hline \multirow[b]{2}{*}{ Categories of Algorithm } & \multirow[b]{2}{*}{$\begin{array}{l}\text { Propagation Speed } \\
\left(\mathrm{m} \cdot \mathrm{s}^{-1}\right)\end{array}$} & \multicolumn{2}{|c|}{ Set 5} & \multicolumn{2}{|c|}{ Set 6} & \multicolumn{2}{|c|}{ Set 7} & \multicolumn{2}{|c|}{ Set 8} \\
\hline & & $\begin{array}{c}x \\
210\end{array}$ & $\begin{array}{c}y \\
210\end{array}$ & $\begin{array}{c}x \\
270\end{array}$ & $\begin{array}{c}y \\
270\end{array}$ & $\begin{array}{c}x \\
450\end{array}$ & $\begin{array}{c}x \\
210\end{array}$ & $\begin{array}{c}x \\
390\end{array}$ & $\begin{array}{c}y \\
270\end{array}$ \\
\hline \multirow{5}{*}{$\begin{array}{c}\text { Delay-and-sum } \\
\text { beamforming [19] }\end{array}$} & 2000 & 210 & 250 & 270 & 260 & 470 & 260 & 390 & 290 \\
\hline & 2500 & 210 & 240 & 270 & 260 & 470 & 230 & 390 & 260 \\
\hline & 3000 & 210 & 240 & 260 & 260 & 460 & 220 & 390 & 260 \\
\hline & 3500 & 210 & 250 & 260 & 260 & 460 & 220 & 390 & 260 \\
\hline & 4000 & 210 & 250 & 260 & 260 & 460 & 220 & 390 & 270 \\
\hline \multirow{9}{*}{ Fast Bartlett beamforming } & 2000 & 210 & 210 & 270 & 270 & 450 & 210 & 390 & 270 \\
\hline & 2500 & 210 & 210 & 270 & 270 & 450 & 210 & 390 & 270 \\
\hline & 3000 & 210 & 210 & 270 & 270 & 450 & 210 & 390 & 270 \\
\hline & 3500 & 210 & 210 & 270 & 270 & 450 & 210 & 390 & 270 \\
\hline & 4000 & 210 & 210 & 270 & 270 & 450 & 210 & 390 & 270 \\
\hline & 6000 & 210 & 210 & 270 & 270 & 450 & 210 & 390 & 270 \\
\hline & 8000 & 210 & 211 & 270 & 270 & 450 & 210 & 390 & 270 \\
\hline & 10,000 & 210 & 208 & 270 & 270 & 450 & 210 & 390 & 270 \\
\hline & 12,000 & 210 & 213 & 270 & 270 & 450 & 210 & 390 & 270 \\
\hline
\end{tabular}




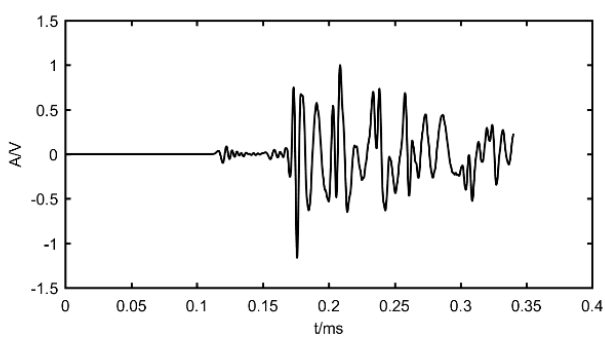

(a)

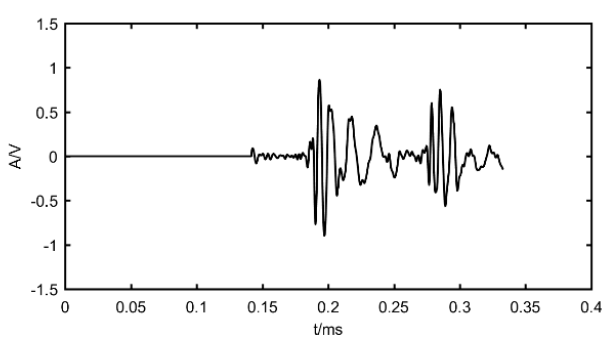

(b)

Figure 10. The comparison of AE signal acquired by simulation and experiment: (a) simulation signal; (b) experimental signal.

Table 5. The average calculation time of two kinds of algorithm with different scanning accuracy.

\begin{tabular}{cccc}
\hline Scanning Accuracy $(\mathbf{m m})$ & Delay-and-Sum Beamforming $(\mathbf{s})$ & Fast Bartlett Beamforming $(\mathbf{s})$ & Ratio \\
\hline 5 & 8.49 & 0.17 & 50.69 \\
2 & 49.16 & 0.35 & 139.96 \\
1 & 193.26 & 0.72 & 269.49 \\
0.5 & 780.19 & 2.13 & 366.50 \\
0.2 & 4887.19 & 11.63 & 420.09 \\
\hline
\end{tabular}

\section{Conclusions}

This paper proposes a fast and reliable algorithm to accurately localize the AE source based on an L-shaped array and Bartlett beamforming. The performance of the algorithm is analyzed by simulation and the PLB test. Some conclusions can be drawn as follows:

(1) Bartlett beamforming cannot be used directly for broadband lamb wave analysis. Based on the presented broadband processing method, the improved Bartlett beamforming has not only great localization performance, but also strong stability at different assumed propagation speeds.

(2) Based on the beam characteristics of Bartlett beamforming, this paper proposes a fast Bartlett beamforming method based on an L-shaped array. According to the simulation result, this method greatly improves the localization efficiency and maintains the accuracy of broadband Bartlett beamforming. Its calculation speed is 224 times faster than that of Bartlett beamforming under the same conditions.

(3) According to the experimental results, the fast Bartlett beamforming method can obtain accurate localization results with a wide range of propagation speed, and its calculation speeds under different scanning grids are 50-420 times faster than delay-and-sum beamforming.

(4) The fast Bartlett beamforming method proposed in this paper has accurate and fast localization performance in a wide range of inaccurate assumed propagation speeds. It is suitable for monitoring and localizing damage sources in large plate structures.

Author Contributions: Conceptualization, T.H. and D.Z.; Methodology, J.T.; Software, D.Z., Q.P.; Validation, J.T., T.H.; Investigation, J.T. and X.W.; Writing-Original Draft Preparation, J.T. and T.H.; Writing-Review \& Editing, X.W.; Funding Acquisition, T.H.

Funding: This research was funded by the National Natural Science Foundation of China (Grant No. 51675023).

Conflicts of Interest: The authors declare no conflict of interest.

\section{References}

1. Mei, H.; Haider, M.F.; Joseph, R.; Migot, A.; Giurgiutiu, V. Recent advances in piezoelectric wafer active sensors for structural health monitoring applications. Sensors 2019, 19, 383. [CrossRef] [PubMed]

2. Sikdar, S.; Banerjee, S. Structural Health Monitoring of Advanced Composites Using Guided Waves; Stefana, G., Ed.; LAP LAMBERT Academic Publishing: Mauritius, East Africa, 2017; ISBN 978-620-2-02697-0. 
3. Kundu, A.; Eaton, M.J.; Al-jumaili, S.K.; Sikdar, S.; Pullin, R. Acoustic emission based damage localization in composites structures using Bayesian identification. J. Phys. Conf. Ser. 2017, 842, 012081. [CrossRef]

4. Sikdar, S.; Ostachowicz, W.; Pal, J. Damage-induced Acoustic Emission Source Identification in an Advanced Sandwich Composite Structure. Compos. Struct. 2018, 202, 860-866. [CrossRef]

5. Sikdar, S.; Mirgal, P.; Banerjee, S.; Ostachowicz, W. Damage-induced acoustic emission source monitoring in a honeycomb sandwich composite structure. Compos. Part B Eng. 2018, 158, 179-188. [CrossRef]

6. Worden, K.; Manson, G.; Allman, D. Experimental validation of a structural health monitoring methodology: Part I. novelty detection on a laboratory structure. J. Sound Vib. 2003, 259, 323-343. [CrossRef]

7. Kundu, T. Acoustic Source Localization. Ultrasonics 2014, 54, 25-38. [CrossRef] [PubMed]

8. Yu, H.S.; Xiao, D.H.; Ma, X.X.; He, T. Near-field beamforming performance analysis for acoustic emission source localization. J. Vibroeng. 2014, 16, 2035-2046.

9. Ziola, S.M.; Gorman, M.R. Source location in thin plates using cross-correlation. J. Acoust. Soc. Am. 1991, 90, 2551-2556. [CrossRef]

10. Hamstad, M.A.; Gallagher, A.O.; Gary, J. Examination of the application of a wavelet transform to acoustic emission signals: Part 2. Source location. J. Acoust. Emiss. 2002, 20, 62-82.

11. Carpinteri, A.; Xu, J.; Lacidogna, G.; Manuello, A. Reliable onset time determination and source location of acoustic emissions in concrete structures. Cem. Concr. Compos. 2012, 34, 529-537. [CrossRef]

12. Kundu, T.; Nakatani, H.; Takeda, N. Acoustic source localization in anisotropic plates. Ultrasonics 2012, 52, 740-746. [CrossRef] [PubMed]

13. Park, W.H.; Packo, P.; Kundu, T. Acoustic source localization in an anisotropic plate without knowing its material properties-A new approach. Ultrasonics 2017, 79, 9-17. [CrossRef] [PubMed]

14. Hensman, J.; Mills, R.; Pierce, S.G.; Worden, K.; Eaton, M. Locating acoustic emission sources in complex structures using gaussian processes. Mech. Syst. Signal Process. 2010, 24, 211-223. [CrossRef]

15. McCrory, J.P.; Al-Jumaili, S.K.; Crivelli, D.; Pearson, M.R.; Eaton, M.J.; Featherston, C.A.; Guagliano, M.; Holford, K.M.; Pullin, R. Damage classification in carbon fibre composites using acoustic emission: A comparison of three techniques. Compos. Part B 2015, 68, 424-430. [CrossRef]

16. McLaskey, G.C.; Glaser, S.D.; Grosse, C.U. Beamforming array techniques for acoustic emission monitoring of large concrete structures. J. Sound Vib. 2010, 12, 2384-2394. [CrossRef]

17. He, T.; Pan, Q.; Liu, Y.G.; Liu, X.D.; Hu, D.Y. Near-field beamforming analysis for AE source localization. Ultrasonics 2012, 5, 587-592. [CrossRef] [PubMed]

18. He, T.; Xie, Y.; Shan, Y.C.; Liu, X.D. Localizing two acoustic emission sources simultaneously using beamforming and singular value decomposition. Ultrasonics 2018, 1, 3-22. [CrossRef] [PubMed]

19. Xiao, D.H.; He, T.; Pan, Q.; Liu, X.D.; Wang, J.; Shan, Y.C. A novel acoustic emission beamforming method with two uniform linear arrays on plate-like structures. Ultrasonics 2014, 54, 737-745. [CrossRef] [PubMed]

20. Huston, R.D. Optimal array configurations for a bartlett beamformer in an arctic environment. J. Acoust. Soc. Am. 1993, 93, 2419. [CrossRef]

21. Liu, X.D.; Xiao, D.H.; Shan, Y.C.; Pan, Q.; He, T. Solder joint failure localization of welded joint based on acoustic emission beamforming. Ultrasonics 2017, 74, 221-232. [CrossRef] [PubMed]

(C) 2019 by the authors. Licensee MDPI, Basel, Switzerland. This article is an open access article distributed under the terms and conditions of the Creative Commons Attribution (CC BY) license (http:// creativecommons.org/licenses/by/4.0/). 